\title{
Solution-sheared thin films of a donor-acceptor random copolymer/polystyrene blend as active material in field-effect transistors
}

By Francesca Leonardi ${ }^{1 \#}$, Qiaoming Zhang ${ }^{1 \#}$, Yun-Hi Kim² and Marta Mas Torrent ${ }^{1 *}$ E-mail: mmas@icmab.es

Affiliations:

${ }^{1}$ Institut de Ciència de Materials de Barcelona (ICMAB-CSIC) and Networking Research Center on Bioengineering, Biomaterials and Nanomedicine (CIBER-BBN), Campus UAB, Bellaterra, 08193 Barcelona Spain

${ }^{2}$ Department of Chemistry and RINS, Gyeongsang, National University Jinju, 660-701, Korea

\#These authors equally contributed to this work

\begin{abstract}
Organic semiconductor (OSC):polymer blends are recently increasing their popularity due to the impressive performances they offer once employed as active layer for organic field-effect transistors (OFETs). Here a novel blend formulation composed by the donor-acceptor random copolymer PDPP-TT(1)-SVS(9) and polystyrene has been processed by a solution shearing technique and employed as active material in a thin film transistor. The molecular weight of the polymer binder has revealed to be fundamental for controlling the vertical phase separation and, in turn, for determining the morphological properties of the upper surface which is critical in terms of device stability. The bi-component active layer has been tested as OFET and by using a top gate electrolyte-gated field-effect transistor (EGOFET) architecture. Our strategy based on an OSC:polymer blend processed through a solution shearing technique has demonstrated to be efficient for improving the final device performance in ambient conditions.
\end{abstract}

\section{Introduction}

In recent years, the use of insulating binders has attracted a growing interest in the field of organic electronics due to the numerous advantages offered by this strategy. ${ }^{1,2}$ For example, the addition of insulating polymers to small molecule organic semiconductors (OSCs) has revealed to be beneficial for facilitating their processability in thin films boosting their exploitation even with many roll-to-roll compatible techniques. ${ }^{3}$ More fundamental studies have also demonstrated how the presence of the insulating polymer promotes crystallinity and homogeneity of the final coating film resulting in an enhancement of the electrical performance. ${ }^{4,5}$ This is mainly due to the vertical phase separation occurring during thin film deposition that promotes the crystallization of the OSC on top of the binder and also often a top thin encapsulation layer of the insulating binder is formed enhancing the material environmental 
stability. ${ }^{6-8}$ This approach has also been explored in polymer OSCs like poly(3-hexylthiophene) (P3HT) improving the stability of the OFET devices ${ }^{9}$ and even allowing the one step deposition of the OSC/dielectric layer. ${ }^{10}$

The organic semiconducting donor-acceptor random copolymer PDPP-TT(1)-SVS(9) (Figure 1) was previously employed as active material in OFETs displaying outstanding performances. ${ }^{11}$ However, the thin films of this material were prepared by spin coating solely under controlled inert atmosphere. In order to assess the potential of this polymer in real applications, here we have adapted a reliable processing procedure, already exploited with many benchmark small molecule OSCs, ${ }^{12,13}$ to this semiconducting polymer. Our work is based on a deposition and characterization protocol carried out under ambient conditions that takes advantage of the following characteristics: i) the blending of the OSC with an insulating polymer, i.e., polystyrene (PS), ii) the thin film deposition by means of a solution shearing technique, i.e. the Bar-Assisted Meniscus Shearing (BAMS), which is compatible with high throughput roll-to-roll processes and iii) the use of a non-chlorinated solvent to deposit the OSC (i.e., tetralin) to realize a fabrication process more environmentally friendly. In the present case, the fabrication approach of our OFETs and EGOFETs resembles the one reported by our group for many benchmarks small molecular OSCs, i.e., dibenzotetrathiafulvalene, ${ }^{14,15}$ TIPS-PEN, DiF-TES$\mathrm{ADT}^{13,16}$ since it has turned out to be a reliable strategy for obtaining high performance devices.

Our results demonstrate that the blend composed by PDPP-TT(1)-SVS(9) and PS can be successfully employed as active material for OFET applications maintaining good performance, even surpassing that of P3HT, one of the most used polymeric OSCs. ${ }^{17}$ Furthermore, this material has been tested using water as dielectric medium exploiting the more challenging architecture called Electrolyte-Gated Field-Effect Transistor (EGOFET) where charge carriers accumulation is modulated by the capacitive coupling of two electrical double layers (EDLs).

\section{Experimental section}

Materials: The organic semiconductor (PDPP-TT(1)-SVS(9)) was obtained by copolymerizing the two repeating units bithiophenyldiketopyrrolopyrrole-selenophene-vinylene-selenophene (DPP-SVS) and bithiophenyldiketopyrrolopyrrole-thienothiophene (DPP-TT) in a TT:SVS ratio of 1:9 according to the synthetic procedure reported by Kim's group. ${ }^{11}$ All the other chemicals were purchased from Sigma Aldrich and used without further purification. During EGOFET measurements, a $0.5 \mathrm{~mm}$ platinum wire (Sigma Aldrich) was employed as gate electrode. QSil216 liquid silicone was purchased from Quantum Silicones and used without any pretreatment. 
Experimental setup: A $200 \mathrm{~nm}$ thick $\mathrm{SiO}_{\mathrm{x}}$ layer thermally growth on a heavily doped n-type $\mathrm{Si}$ (Si-Mat) represents the gate dielectric/gate electrode stack of the OFET device. Gold source $(S)$ and drain $(D)$ electrodes were pre-patterned on $\mathrm{SiO}_{\mathrm{x}} / \mathrm{Si}$ substrates by photolithography (Micro Writer ML3 from Durham Magneto Optics Ltd.) followed by the metal evaporation (system Auto 306 from Boc Edwards) of Cr/Au layer (5/40 nm). The channel width/length $(W / L)$ ratio was fixed to 690 (i.e., 20700 and $30 \mu \mathrm{m}$ as $W$ and $L$, respectively). EGOFETs were measured by means of a Pt wire immersed in MilliQ water confined on the top of the device with a PDMS pool. The electrochemical measurements were realized on $\mathrm{SiO}_{\mathrm{x}} / \mathrm{Si}$ substrates uniformly coated with a $\mathrm{Cr} / \mathrm{Au}$ layer $(5 / 40 \mathrm{~nm})$.

Prior to organic material deposition, substrates were cleaned in ultrasonic bath with acetone and isopropanol for 15 minutes each and subsequently ozone-treated for 25 minutes. Afterwards, in order to improve the coating adhesion during the deposition of the organic material, gold electrodes were modified with a self-assembled monolayer of pentafluorothiophenol (PFBT) by immersion in a $15 \mathrm{mM}$ solution of PFBT in isopropanol.

After preliminary tests for thin film deposition varying the OSC:PS ratio from 1:2 to 1:9, the coating speed from 0.1 to $2 \mathrm{~cm} / \mathrm{s}$ and the temperature (i.e., 105 and $150{ }^{\circ} \mathrm{C}$ ), we observed that the best performances were obtaining selecting the following conditions to prepare the thin films (Figure S1). The OSC:PS blend solution was prepared in a 1:2 volume ratio (1 wt\%) using tetralin as solvent. The solution was kept on a hot plate at $105^{\circ} \mathrm{C}$ for more than 30 minutes to ensure the complete dissolution of the material. Polystyrene with two different molecular weights, i.e., $\mathrm{Mw} 3.000 \mathrm{~g} \mathrm{~mol}^{-1}$ (PS3K) and $100.000 \mathrm{~g} \mathrm{~mol}^{-1}$ (PS 100K), were used as insulating binders for the preparation of PDPP-TT(1)-SVS(9):PS blends. The blend coating was realized in ambient conditions using Bar-Assisted Meniscus Shearing (BAMS) technique at a speed of 1 $\mathrm{cm} / \mathrm{s}$ and keeping the supporting plate at a constant temperature of $150^{\circ} \mathrm{C}$.

Electrical measurements have been performed with an Agilent 5100A equipped with EasyExpert software. Atomic Force Microscopy (AFM) images were acquired with 5500LS SPM system from Agilent Technologies and analyzed with Gwyddion 2.48 software. Electrochemical Impedance Spectroscopy (EIS) experiments were performed using a Novocontrol Alpha-AN impedance analyzer equipped with POT/GAL 30 V/2 A electrochemical interface. EIS and cyclic voltammetry (CV) experiments have been performed on a Pt/water/OSC:PS blend/Au structure and on $\mathrm{Pt} /$ water/Au who serves as reference measurement. The Pt electrode and the gold substrate $\left(0.18 \mathrm{~cm}^{2}\right)$ were used as counter and working electrodes, respectively. Impedance spectra were recorded in a $10^{-1} / 10^{5} \mathrm{~Hz}$ frequency range by applying a $V_{A C}$ of $0.02 \mathrm{mV}$ sine-wave voltage signal. Two $\mathrm{DC}$ bias ( $0 \mathrm{~V}$ and $-0.6 \mathrm{~V}$ ) were superimposed to the AC signal. Data analysis was performed with NOVA 2.1 software 
Chemical composition of the OSC was detected by ToF-SIMS, surface sputter etching was accomplished with $\mathrm{O}_{2}$ beam, over a $300 \mu \mathrm{m} \times 300 \mu \mathrm{m}$ area using $1 \mathrm{keV}$ energy settings raster. A pulsed beam of $25 \mathrm{keV} \mathrm{Bi}_{3}{ }^{++}$ions scanned over a $50 \mu \mathrm{m} \times 50 \mu \mathrm{m}$ region centered within the sputtered area was used. Analysis cycle time was $100 \mu$ s and sputtering time for each cycle was $1 \mathrm{~s}$. In addition, the X-ray diffraction measurement was performed with a diffractometer from Rigaku, equipped with a rotating anode source.

\section{Results and discussion}

Nowadays, solution shearing techniques represent a widely used approach for the deposition of organic thin films. These techniques have revealed to be fundamental for puzzling out many structure-properties relationship issues of organic electronic devices. ${ }^{18}$

As mentioned in the introduction, PDPP-TT(1)-SVS(9) has already displayed excellent performance as active materials for OFET. However, our work deals with a technique compatible with up-scaling and the fabrication process has been entirely performed under environmental conditions. Remarkably, the OSC:PS blend has been processed from tetralin solutions, avoiding the use of potentially harmful chlorinated solvents frequently used with organic semiconductors.

The role of polystyrene is pivotal for the formation of compact and homogeneous thin film and the molecular weight of the insulating polymer has demonstrated to be fundamental in determining the degree of thin film crystallinity and, in turn, the final device performance. ${ }^{19}$ Therefore, two polystyrene of different molecular weights, i. e. $3 \mathrm{~K}$ and $100 \mathrm{~K}$, have been chosen as insulating binders for PDPP-TT(1)-SVS(9) and they have been compared with the pristine OSC thin film. All the films used for the OFET and EGOFETs fabrication were prepared on $\mathrm{Si} / \mathrm{SiO}_{\mathrm{x}}$, as displayed in Figure 1a. In order to evaluate the vertical phase separation, the films were characterized by ToF-SIMS, which seem to indicate that the OSC PDPP-TT(1)-SVS(9) was mainly located on the bottom part of the film (Figure S2).

As evidenced in Figure $1 \mathrm{~b}$ and d, the OFET performance of the two kinds of devices displays a clear p-type behavior with the PS3K-based blend surpassing the electrical results obtained from the device based on PDPP-TT(1)-SVS(9):PS100k one (Fig. S3a, c). The threshold voltage ( $\left.V_{t h}\right)$ and hysteresis are remarkably more ideal in the case of PDPP-TT(1)-SVS(9):PS3K blend compared to the PDPP-TT(1)-SVS(9):PS100K one, which probably indicates a lower trap density at the $\mathrm{OSC} / \mathrm{SiO}_{\mathrm{x}}$ interface in the PDPP-TT(1)-SVS(9):PS3K case. The charge carrier mobility $(\mu), V_{\text {th }}$ and on/off ratio $\left(I_{o n} / I_{\text {off }}\right)$ extracted from the electrical characteristics are listed in Table S1. The overall charge-carrier mobility remains lower compared to the one previously reported by Kim et al. ${ }^{11,20}$ which is an expected consequence considering that our thin film processing and electrical measurements are performed under ambient conditions and, in contrast 
to the previous work, no temperature post-annealing step was performed here to achieve thin film crystallinity. However, remarkable mobilities are still achieved, especially considering that the as-prepared films are amorphous (see Figure S4 for XRD data), being the average $\mu$ of PS 3K and PS 100K-based blends $0.22 \mathrm{~cm}^{2} \mathrm{~V}^{-1} \mathrm{~s}^{-1}$ and $0.10 \mathrm{~cm}^{2} \mathrm{~V}^{-1} \mathrm{~s}^{-1}$, respectively. Unfortunately, the subsequent annealing of the blend films revealed to be detrimental for the electrical performance (Figure S5).

It should be highlighted that thin films prepared following the same methodology but using solely PDPP-TT(1)-SVS(9) exhibit poor electrical performance, as reported in Figure S6a,c. Low $I_{D S}$ and a huge hysteresis were observed. This can be ascribed to the poor film formation due to the lower viscosity of the solution used. Indeed, this parameter is fundamental when solution shearing techniques are employed, particularly at high coating speeds. ${ }^{19}$ Further, the use of PS has previously been shown to be useful for passivating the hydroxyl groups present in the $\mathrm{SiO}_{2}$ dielectric that act as charge traps, which results in devices with less hysteresis and steeper switch on. ${ }^{21,22}$ Thus, all this elucidates the important role of the binding polymer for processing OSCs. However, it should be mentioned that although the use of PS is pivotal to be able to process the OSC in environmental conditions, it does not represent an impermeable barrier in front of oxygen and moisture. Therefore, for the fabrication of devices with long-term stability an additional encapsulation layer should be required for these films. 


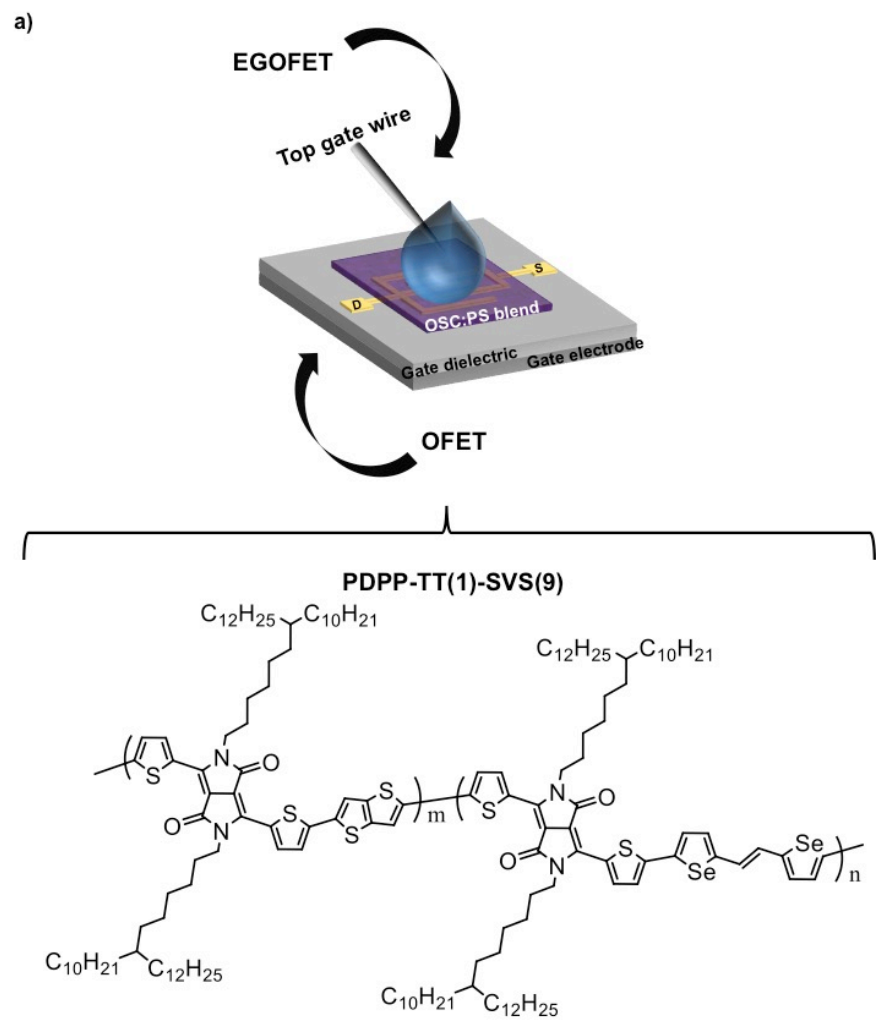

PS
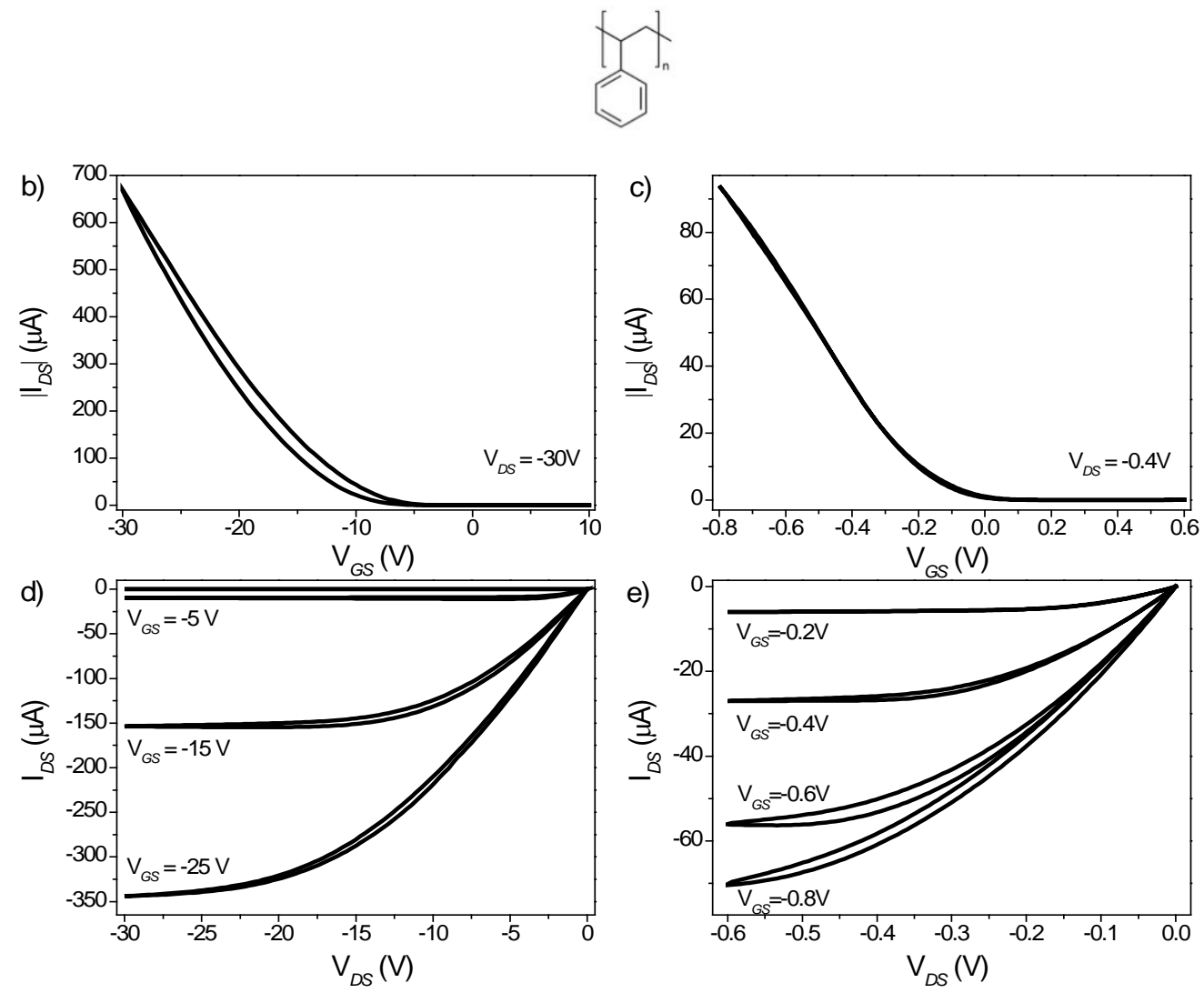

Figure 1 a) Schematic diagram of the OFET/EGOFET setup and the chemical structure of the organic semiconductor PDPP-TT(1)-SVS(9). Electrical performance of PDPP-TT(1)- 
SVS(9):PS3K measured as OFET (b and d) and as EGOFET device (c and e) using MilliQ water as electrolyte.

By switching to top gate architecture and to a liquid medium, EGOFET results show once again the superior performance of the PS3K-based devices. As shown in Figure 1c and e, the representative $I$ - $V$ output and transfer curves of the EGOFET based on this formulation are free of any hysteresis and possess state-of-the-art values of on/off ratio $\left(\sim 10^{4}\right)$. For comparison, the electrical performance of PDPP-TT(1)-SVS(9):PS100K are also reported in Figure S3b and d. Although no visible hysteresis was observed in the PDPP-TT(1)-SVS(9):PS100K case, it exhibited a lower electrical performance, i.e., less on current and ON/OFF ratio. It is worth mentioned that the leakage current in both two cases are several tens of $\mathrm{nA}$, which is 2 or 3 order of magnitudes lower than the source-drain current in each case (Figure S7). In addition, no direct influence of the leakage current on the ON/OFF ratio was observed. The $\mu \cdot C_{s a t}, V_{t h}$ and $I_{\text {on }} / I_{\text {off }}$ of the two blends in the saturation regime are summarized in Table S1. The product of $\mu \cdot C_{\text {sat }}$ has been selected as figure of merit to describe the charge carrier accumulation process, reaching average values of 0.15 and $0.33 \mu \mathrm{F} \mathrm{V} \mathrm{V}^{-1} \mathrm{~s}^{-1}$ for the PS100K and PS3k blends, respectively. Also in this case, the devices prepared without PS revealed a much lower performance (Figure S6b,d), with $\mu \cdot C_{\text {sat }}$ values of one order of magnitude lower $\left(0.02 \mu \mathrm{F} \mathrm{V} \mathrm{V}^{-1} \mathrm{~s}^{-}\right.$ ${ }^{1}$ ), proving again that the insulating binder plays a key role for achieving high electrical performance also in this device architecture.

One of the most straightforward features regarding EGOFET technology is the necessity of a robust and stable operation in water media. Thus, understanding the processes occurring at the electrolyte/OSC interface is of outmost importance. Previous studies already demonstrated how polystyrene play a fundamental role for conferring water stability to several organic semiconductors ${ }^{15}$ even if a certain degree of porosity and permeation is present. ${ }^{23}$ Despite many issues have already been addressed, many murky points regarding the role of the insulating binder still need to be clarified. In order to understand this quite marked difference of the electrical performances, we have carried on an electrochemical and morphological study on thin films composed by PDPP-TT(1)-SVS(9) and polystyrene of the two different molecular weights, i.e., $3 \mathrm{~K}$ and $100 \mathrm{~K}$.

Electrochemical impedance spectroscopy (EIS) is a powerful tool for investigating the OSC/electrolyte interface. A stacked architecture comprising of Pt/water/ PDPP-TT(1)SVS(9):PS/Au has been studied in the frequency range spanning from $10^{5}$ to $10^{-1} \mathrm{~Hz}$. Taking into account the operational window of the EGOFET, two DC voltages, i.e., $0 \mathrm{~V}$ and $-0.6 \mathrm{~V}$, were selected and superposed to an AC bias of $20 \mathrm{mV}$ amplitude. 
Figure 2 shows the Bode plots recorded in MilliQ water for both PDPP-TT(1)-SVS(9):PS3K and PDPP-TT(1)-SVS(9):PS100K blends. At $0 \mathrm{~V}$, the thin films prepared with PS3K and PS100K display a similar phase $(\theta)$ response, however, PS100K displayed higher impedance as depicted in Figure 2a and c. At high frequency (HF, $f>100 \mathrm{~Hz}$ ) the two systems display a conductive behavior, which is probably due to the polarization of water and the absence of a supporting electrolyte. However, the blend-coated sample evidenced $\theta$ values closer to $30^{\circ}$ at $f>$ $10^{4} \mathrm{~Hz}$ deviating from the pure conducting behavior observed in the uncoated sample (see Fig. S8 in Supporting Information). As reported by Cotrone et al., ${ }^{24}$ this $\theta$ response could be ascribed to the migration of water ions through the thin film. In the low frequency regime (LF, $f<100$ $\mathrm{Hz}$ ), the system responds as a capacitor due to the formation of a Helmholtz double layer. By switching the DC voltage to $-0.6 \mathrm{~V}$, the impedance of the two films decrease and the phase response remains unchanged except at LF regime where a predominantly more conductive response is observed $\left(0<\theta<45^{\circ}\right)$. A similar DC dependence was already reported by other authors $^{25,26}$ and it is probably due to the onset of water electrolysis or to a charge transfer process related to the presence of the organic semiconductor. ${ }^{27}$ 

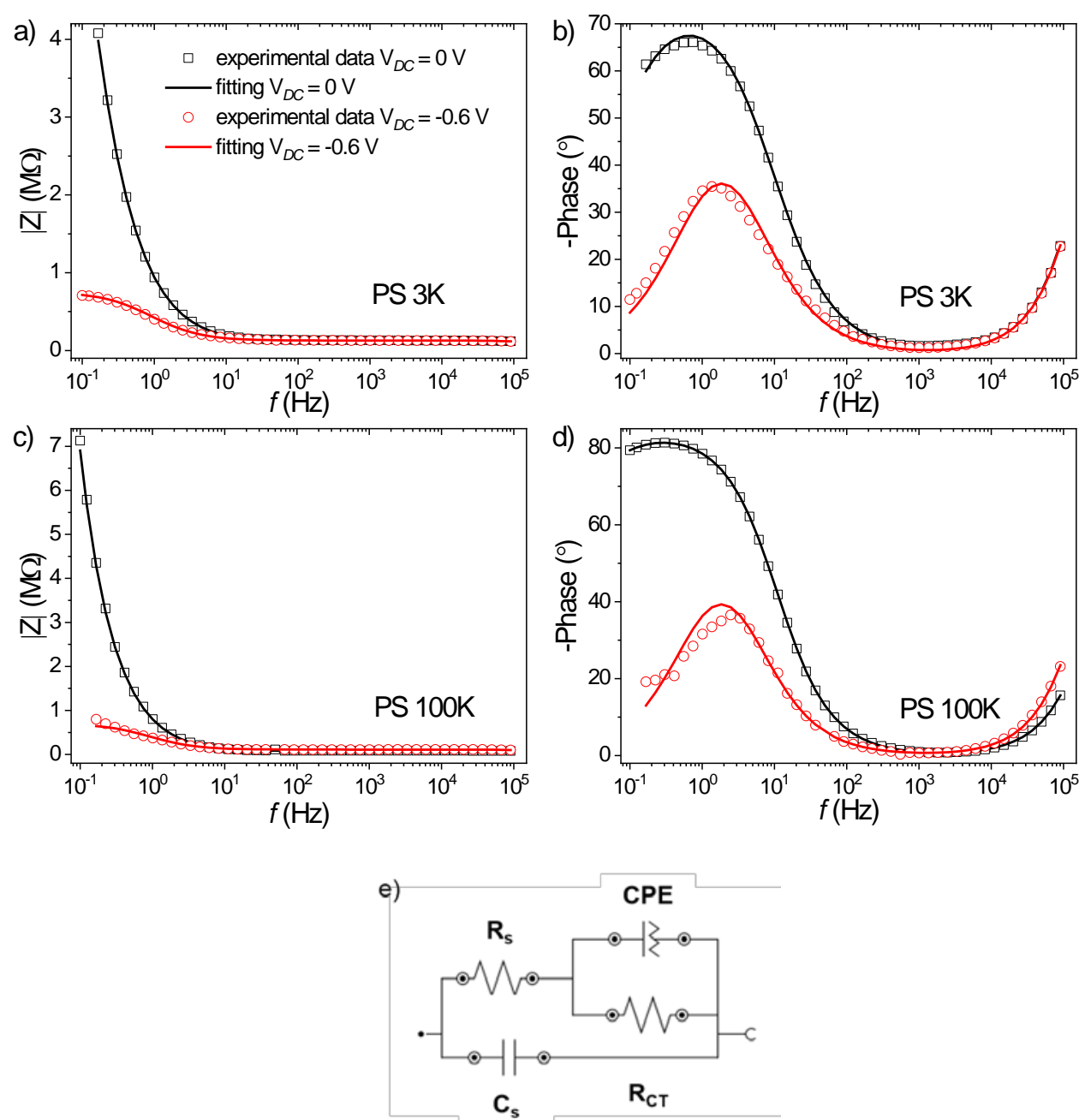

Figure 2 (a and c) Impedance and (b and d) phase angle response of the PDPP-TT(1)SVS(9):PS3K and PDPP-TT(1)-SVS(9):PS100K blend films at two selected DC voltages. The open symbols represent the experimental data while the solid lines correspond to the fitting results. e) Equivalent circuit employed for data fitting: $R_{s}=$ solution resistance, $C_{s}=$ geometrical capacitance, $R_{C T}$ charge transfer resistance, $\mathrm{CPE}=$ Constant Phase Element.

The impedance behavior of the metal/water/ PDPP-TT(1)-SVS(9):PS/metal was described by the equivalent circuit shown in Figure 2e. ${ }^{24,26,28,29}$ A first RC circuit describes the water medium behavior at high frequency ( $f>100 \mathrm{~Hz}$ ) and $R_{S}$ and $C_{S}$ represents the resistance and the geometrical capacitance of the electrolytic solution, respectively. The second RC, which accounts for the double layer formation at low frequency region $(<100 \mathrm{~Hz})$, includes the contribution of the resistance to charge transfer process $\left(R_{C T}\right)$ and of the Helmholtz double-layer capacitance $\left(C_{D L}\right)$. In order to account for the surface heterogeneities, $C_{D L}$ has been replaced by 
a Constant Phase Element (CPE) which is commonly represented as $Z(\omega)=\left(1 / Q_{0}(j \omega)^{n}\right)$. All the parameters obtained from the fitting procedure have been listed in Table 1.

For values of $n$ close to unity, $Q_{0}$ represents a capacitance so we have extracted the effective capacitance according to: ${ }^{30}$

$C_{D L, e f f}=Q_{0}^{\frac{1}{n}} R_{C T}^{\left(\frac{1}{n}\right)-1}$

An abrupt decrease of $R_{C T}$ (over 2 orders of magnitude) is observed at a DC potential equal to $-0.6 \mathrm{~V}$, which could be ascribed to the polarization of the OSC layer and the further penetration of ions inside the thin film. This trend is accompanied by an increase of $C_{D L \text {,eff }}$ that can also be attributed to the charging of the OSC. The comparison of the fitting results with the values of the double layer capacitance $\left(C_{D L, \omega}\right)$ extracted at a frequency of $10 \mathrm{~Hz}$ confirms the quality of our method. In addition, the value of $C_{D L, \omega}$ are in line with values reported for polymer OSC $^{25}$ but one order of magnitude lower compared to the values reported for DBTTF, TIPS-PEN and diF-TES-ADT, which are high crystalline small-molecule OSC. ${ }^{15,16}$

Table 1 Fitting values of the EIS experimental data.

\begin{tabular}{|c|c|c|c|c|}
\hline & \multicolumn{2}{|c|}{ PDPP-TT(1)-SVS(9):PS3K } & \multicolumn{2}{|c|}{ PDPP-TT(1)-SVS(9):PS100K } \\
\hline & $\mathrm{V}_{\mathrm{DC}}=0 \mathrm{~V}$ & $V_{D C}=-0.6 \mathrm{~V}$ & $\mathrm{~V}_{\mathrm{DC}}=0 \mathrm{~V}$ & $\mathrm{~V}_{\mathrm{DC}}=-0.6 \mathrm{~V}$ \\
\hline $\mathrm{R}_{\mathrm{s}}(\mathrm{M} \Omega)$ & 0.13 & 0.13 & 0.08 & 0.11 \\
\hline $\mathrm{C}_{\mathrm{s}}(\mathrm{pF})$ & 5.78 & 5.77 & 5.9 & 6.9 \\
\hline $\mathrm{R}_{\mathrm{CT}}(\mathrm{M} \Omega)$ & 9.08 & 0.58 & 66.1 & 0.54 \\
\hline$Q_{0}$ (nS s) & 196 & 429 & 218 & 471 \\
\hline$n(-)$ & 0.923 & 0.883 & 0.951 & 0.901 \\
\hline $\mathrm{C}_{\mathrm{DL}, \text { eff }}(\mathrm{nF})$ & 20.7 & 57.2 & 60.2 & 88.7 \\
\hline $\mathrm{C}_{\text {DL }, \text { eff }}^{\prime}\left(\boldsymbol{\mu} \mathbf{F} \mathbf{~ c m}^{-2}\right)$ & 0.11 & 0.32 & 0.33 & 0.49 \\
\hline$C_{D L, \omega}\left(\mu \mathrm{F} \mathrm{cm} \mathbf{c m}^{-2}\right)$ & 0.25 & 0.25 & 0.26 & 0.34 \\
\hline
\end{tabular}

At last, the morphological differences of the two blends were evaluated by AFM. The thickness of the two films is similar, $26.5 \mathrm{~nm}$ and $31.5 \mathrm{~nm}$ for PDPP-TT(1)-SVS(9):PS3K and PDPPTT(1)-SVS(9):PS100K, respectively (Figure S9). The images seem to suggest that a thin PS layer is lying on top of the polymeric semiconductor. This phenomenon has already been observed for different small molecule OSCs. ${ }^{8}$

Figure 3a corresponds to PDPP-TT(1)-SVS(9):PS3K blend and it reveals a non-homogeneous surface (Root Mean Square roughness, rms, $\delta=5.7 \mathrm{~nm}$ ) possibly dictated by the present of PS as top layer. However, the phase image (Figure S10) revealed a complex morphology, which could be characteristic of the OSC layer below. For reference, the AFM images of thin films of the pristine OSC are shown in Figure S11, where a clear fibrillar microstructure can be observed. In the case of PDPP-TT(1)-SVS(9):PS100K (rms, $\delta=5.4 \mathrm{~nm}$ ) the scenario is 
markedly different. The top PS layer seems thicker compared to the previous case likely caused by the higher viscosity of the film. As a consequence, the phase image cannot reveal any detail of the bottom OSC. Noticeably, numerous holes (depth $=17.1 \pm 1.2 \mathrm{~nm}$ ) are observed which allow a glimpse of the OSC layer underneath (Figure 3b). The influence of the solution viscosity on the thin film homogeneity has already been previously elucidated. ${ }^{18,19}$ The thicker PS top layer in the case of PDPP-TT(1)-SVS(9):PS100K and the hydrophobic nature of the coating could account of the huge $\mathrm{R}_{\mathrm{CT}}$ calculated from EIS measurements at $\mathrm{V}_{\mathrm{DC}}=0 \mathrm{~V}$, however, it is reasonable to believe that the numerous holes of the top layer could facilitate the penetration of the electrolyte at a $\mathrm{V}_{\mathrm{DC}}=-0.6 \mathrm{~V}$ due to the polarization of the OSC explaining the dramatic drop of this value. Further, we believe that the fact that the PS3K films are more homogenous with less holes might also account for the improved device performance achieved in these films when measured in ambient conditions.

Considering all above, we can affirm that the presence of PS during the deposition of PDPPTT(1)-SVS(9) facilitates the processing of this material in ambient conditions, since it acts as an encapsulation layer due to the vertical phase separation taking place during the deposition. This permits the benchtop fabrication of remarkably performing OFETs and EGOFETs.

a)

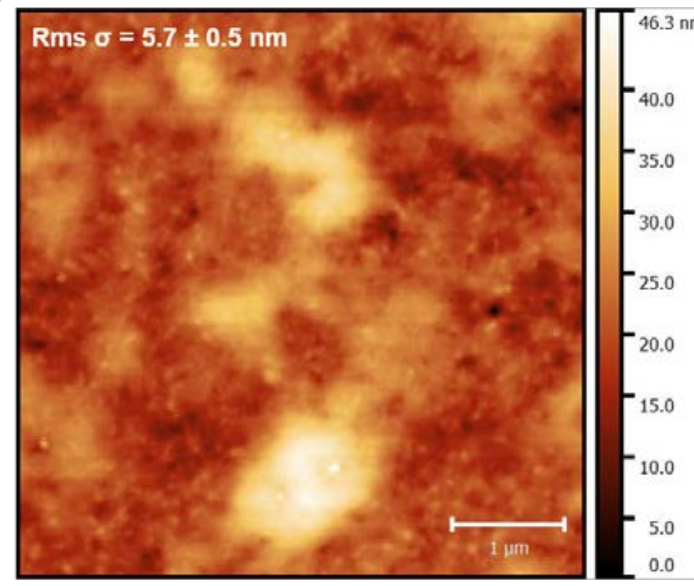

b)

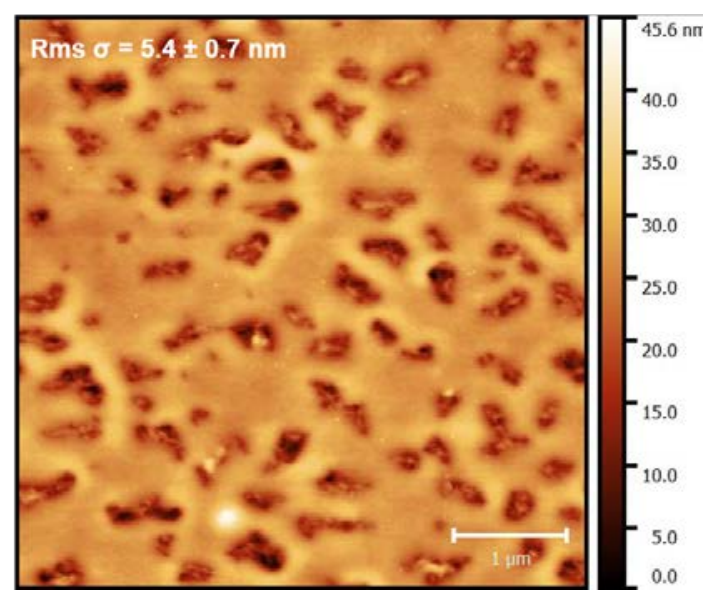

Figure 3 AFM images $\left(5 \times 5 \mathrm{~m}^{2}\right)$ of (a) PDPP-TT(1)-SVS(9):PS3K and (b) PDPP-TT(1)SVS(9):PS100K. The rms values are averaged from 5 images.

\section{Conclusion}

In conclusion a new polymer OSC:PS blend have been successfully characterized in both OFET and EGOFET configurations. This OSC, namely PDPP-TT(1)-SVS(9), has already been reported to display excellent electrical characteristics, although its processing and characterization were restricted to a controlled inert atmosphere. Our results demonstrated how the approach including the use of an OSC:polymer blend as starting material and a solution shearing technique, i.e., BAMS, can allow the control of the thin film properties and limit the 
electrical degradation of the device. Moreover, in the present case the use of chlorinated solvents has been avoided and a greener option, i.e., tetralin, was the selected solvent.

Both OFET and EGOFET devices based on blends have displayed significantly improved performances with respect to the devices prepared without the binding polymer, with the PDPPTT(1)-SVS(9):PS3K based-film showing a superior performance than the PDPP-TT(1)SVS(9):PS100K one in both configurations. These results confirmed the impact of using polystyrene and the influence of its molecular weight on the electrical performance. The electrochemical impedance results have confirmed the insulating character of our thin films at a potential of $0 \mathrm{~V}$ while at $-0.6 \mathrm{~V}$ the phase angle deviates from the ideal LF behavior and a more conductive character appears. Further, AFM has evidenced how the molecular weight of the insulating polymer is crucial during the vertical phase separation for the formation of a continuous top encapsulating layer. Although in PDPP-TT(1)-SVS(9):PS100K films a thicker top PS layer is formed, the numerous porous found on this top surface seems to be a direct consequence of the increased viscosity of this blend solution. This study has elucidated the pivotal role of using binding polymers with promising high-mobility OSCs to confer environmental stability and, hence, realize devices with higher potential for low-cost applications.

\section{Acknowledgements}

F.L. and Q.Z. contributed equally to this work. F.L., Q.Z. and M.M.-T. thank the ERC-StG 2012-306826 e-GAMES project, the Networking Research Center on Bioengineering, Biomaterials and Nanomedicine (CIBER-BBN), the DGI (Spain) project, FANCY CTQ201680030-R, the Generalitat de Catalunya (2017-SGR-918), and the Spanish Ministry of Economy and Competitiveness, through the "Severo Ochoa" Program for Centres of Excellence in R\&D (SEV-2015-0496). Q.Z. acknowledges the China Scholarship Council, the National Natural Science Foundation (NSF) of China (Grant No. 11404266). Q.Z. was enrolled in the Materials Science PhD Program of Universitat Autònoma de Barcelona. F.L. gratefully acknowledges the “Juan de la Cierva” program. Y.-H. K. Thanks the National Research Foundation of Korea (NRF) funded by the Ministry of Science (NRF-2015R1A2A1A10055620).

\section{References}

1 C. Teixeira da Rocha, K. Haase, Y. Zheng, M. Löffler, M. Hambsch and S. C. B. Mannsfeld, Adv. Electron. Mater., 2018, 1800141, 1-9. 
C. E. Murphy, L. Yang, S. Ray, L. Yu, S. Knox and N. Stingelin, J. Appl. Phys., 2011, 110, 93523.

Y. Lei, P. Deng, M. Lin, X. Zheng, F. Zhu and B. S. Ong, Adv. Mater., 2016, 28, 66876694.

M. Lada, M. J. Starink, M. Carrasco, L. Chen, P. Miskiewicz, P. Brookes, M. Obarowska and D. C. Smith, J. Mater. Chem., 2011, 21, 11232-11238.

X. Wang, W. H. Lee, G. Zhang, X. Wang, B. Kang, H. Lu, L. Qiu and K. Cho, J. Mater. Chem. C, 2013, 1, 3989.

Y. H. Kim, J. E. Anthony and S. K. Park, Org. Electron., 2012, 13, 1152-1157.

A. Pérez-Rodríguez, I. Temiño, C. Ocal, M. Mas-Torrent and E. Barrena, ACS Appl. Mater. Interfaces, 2018, 10, 7296-7303.

A. C. Arias, F. Endicott and R. A. Street, Adv. Mater., 2006, 18, 2900-2904.

L. Qiu, J. A. Lim, X. Wang, W. H. Lee, M. Hwang and K. Cho, Adv. Mater., 2008, 20, 1141-1145.

11 H. J. Yun, J. Cho, D. S. Chung, Y. H. Kim and S. K. Kwon, Macromolecules, 2014, 47, 7030-7035.

12 A. Campos, S. Riera-Galindo, J. Puigdollers and M. Mas-Torrent, ACS Appl. Mater. Interfaces, 2018, 10, 15952-15961.

13 I. Temiño, F. G. Del Pozo, M. R. Ajayakumar, S. Galindo, J. Puigdollers and M. MasTorrent, Adv. Mater. Technol., 2016, 1, 1600090.

14 F. G. del Pozo, S. Fabiano, R. Pfattner, S. Georgakopoulos, S. Galindo, X. Liu, S. Braun, M. Fahlman, J. Veciana, C. Rovira, X. Crispin, M. Berggren and M. Mas-Torrent, Adv. Funct. Mater., 2015, 26, 2379-2386.

15 F. Leonardi, S. Casalini, Q. Zhang, S. Galindo, D. Gutiérrez and M. Mas-Torrent, Adv. Mater., 2016, 28, 10311-10316.

16 Q. Zhang, F. Leonardi, S. Casalini, I. Temiño and M. Mas-Torrent, Sci. Rep., 2016, 6, 39623.

17 S. Vegiraju, C. Y. Lin, P. Priyanka, D. Y. Huang, X. L. Luo, H. C. Tsai, S. H. Hong, C. J. Yeh, W. C. Lien, C. L. Wang, S. H. Tung, C. L. Liu, M. C. Chen and A. Facchetti, Adv. Funct. Mater., 2018, 1801025, 1-10.

18 S. Riera-Galindo, A. Tamayo and M. Mas-Torrent, ACS Omega, 2018, 3, 2329-2339.

19 M. R. Niazi, R. Li, E. Qiang Li, A. R. Kirmani, M. Abdelsamie, Q. Wang, W. Pan, M. M. Payne, J. E. Anthony, D.-M. Smilgies, S. T. Thoroddsen, E. P. Giannelis and A. Amassian, Nat. Commun., 2015, 6, 8598. 

6612-6616.

21 A. Campos, S. Riera-Galindo, J. Puigdollers and M. Mas-Torrent, ACS Appl. Mater. Interfaces, 2018, 10, 15952-15961.

22 A. Campos, Q. Zhang, M. R. Ajayakumar, F. Leonardi and M. Mas-Torrent, Adv. Electron. Mater., 2017, 1700349.

23 Q. Zhang, F. Leonardi, S. Casalini and M. Mas-Torrent, Adv. Funct. Mater., 2017, 27, 1703899 .

24 S. Cotrone, M. Ambrico, H. Toss, M. D. Angione, M. Magliulo, A. Mallardi, M. Berggren, G. Palazzo, G. Horowitz, T. Ligonzo and L. Torsi, Org. Electron., 2012, 13, 638-644.

25 R. Porrazzo, S. Bellani, A. Luzio, E. Lanzarini, M. Caironi and M. R. Antognazza, Org. Electron., 2014, 15, 2126-2134.

26 L. Kergoat, L. Herlogsson, D. Braga, B. Piro, M.-C. Pham, X. Crispin, M. Berggren and G. Horowitz, Adv. Mater., 2010, 22, 2565-9.

27 G. Tullii, A. Desii, C. Bossio, S. Bellani, M. Colombo, N. Martino, M. R. Antognazza and G. Lanzani, Org. Electron. physics, Mater. Appl., 2017, 46, 88-98.

F. Buth, D. Kumar, M. Stutzmann and J. A. Garrido, Appl. Phys. Lett., 2011, 98, 153302.

29 C. Suspène, B. Piro, S. Reisberg, M. C. Pham, H. Toss, M. Berggren, A. Yassar and G. Horowitz, J. Mater. Chem. B, 2013, 1, 2090-2097.

30 A. J. Bard and L. R. Faulkner, Electrochemical methods, Fundamentals and Applications, John Wiley \& Sons, Inc., New York., 2001. 
Supporting information
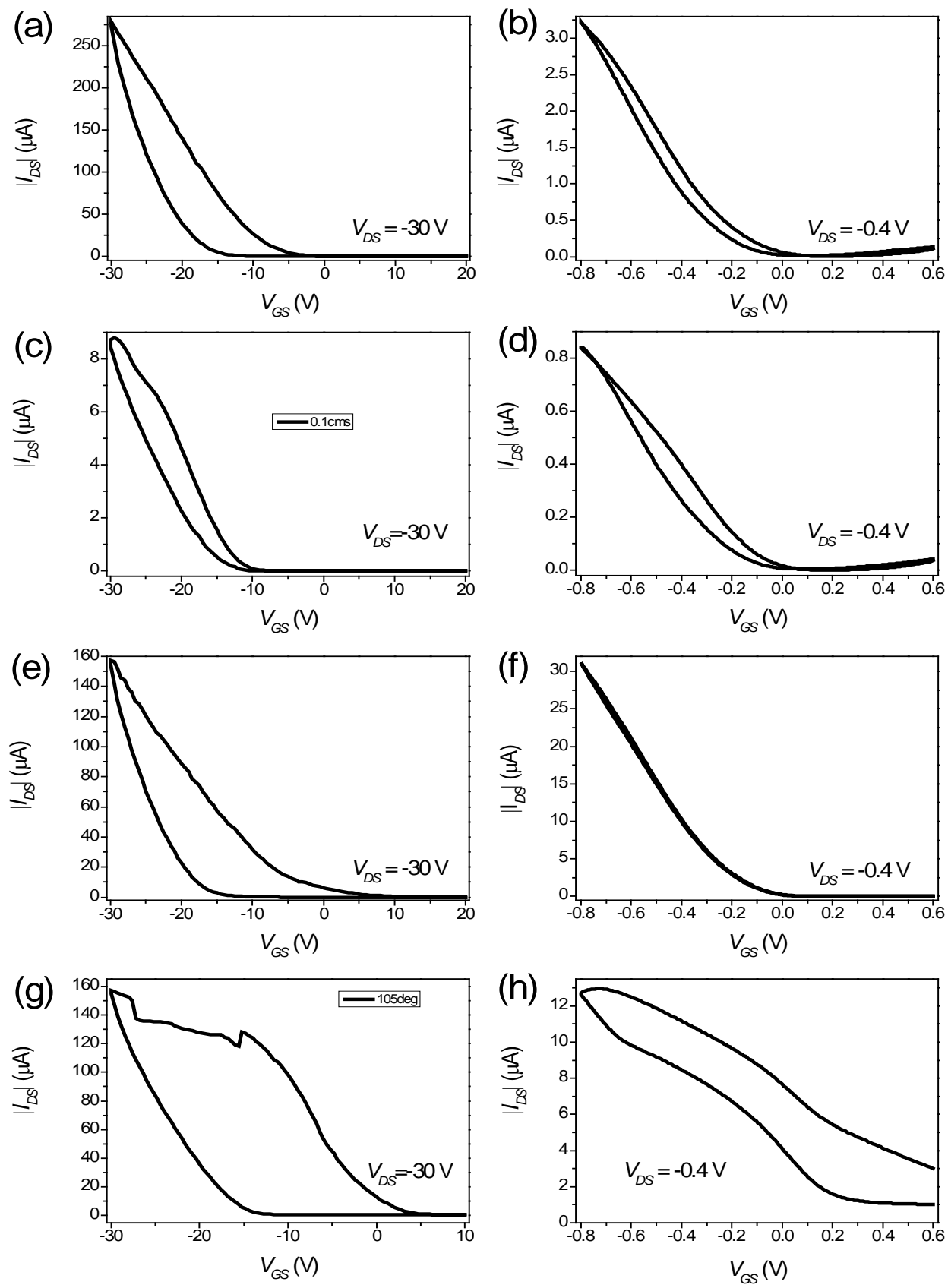

Figure S1 Electrical performance of PDPP-TT(1)-SVS(9):PS3K blend measured by means of OFET (left) and EGOFET (right) configuration according to different ink formulations and different coating parameters. (a/b) OSC:polymer ratio 1:9; coating speed $=1 \mathrm{~cm} / \mathrm{s}$; coating temperature $=150{ }^{\circ} \mathrm{C}$. (c/d) OSC:polymer ratio $1: 2$; coating speed $=0.1 \mathrm{~cm} / \mathrm{s}$; coating temperature $=150{ }^{\circ} \mathrm{C}$. (e/(f) OSC:polymer ratio 1:2; coating speed $=2 \mathrm{~cm} / \mathrm{s}$; coating temperature $=150{ }^{\circ} \mathrm{C}$. $(\mathrm{g} / \mathrm{h})$ OSC:polymer ratio $1: 2$; coating speed $=1 \mathrm{~cm} / \mathrm{s}$; coating temperature $=105^{\circ} \mathrm{C}$. 


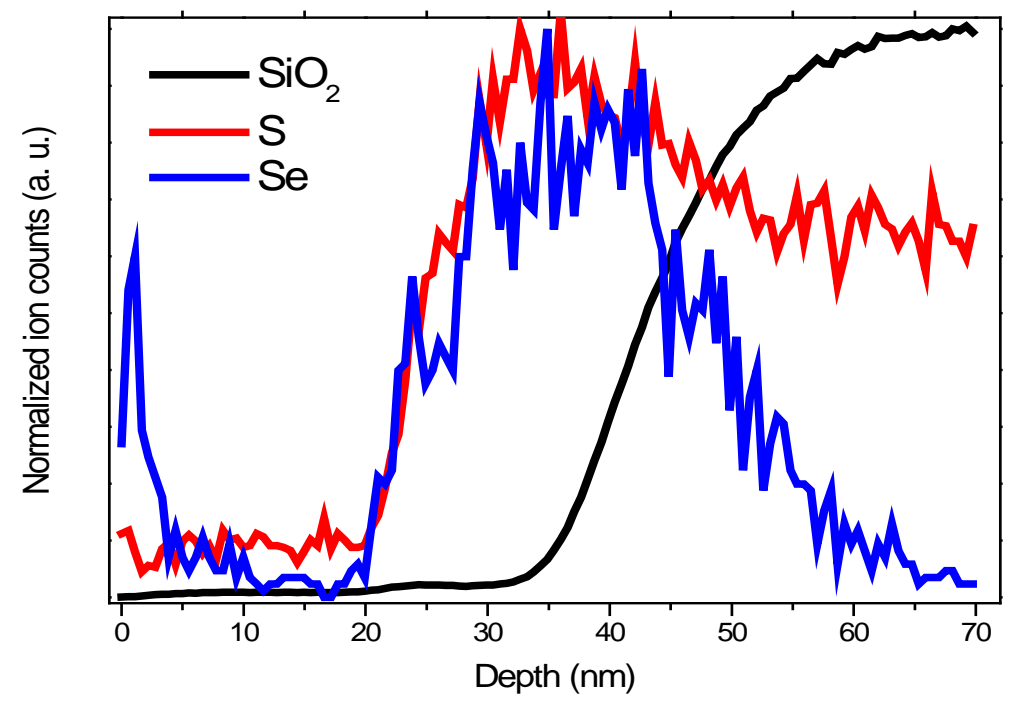

Figure S2 ToF-SIMS depth profile of $\mathrm{SiO}_{2}$, S, and Se of a typical of PDPP-TT(1)-SVS(9):PS thin film starting from the surface and reaching the $\mathrm{SiO}_{2} /$ organic blend interface.
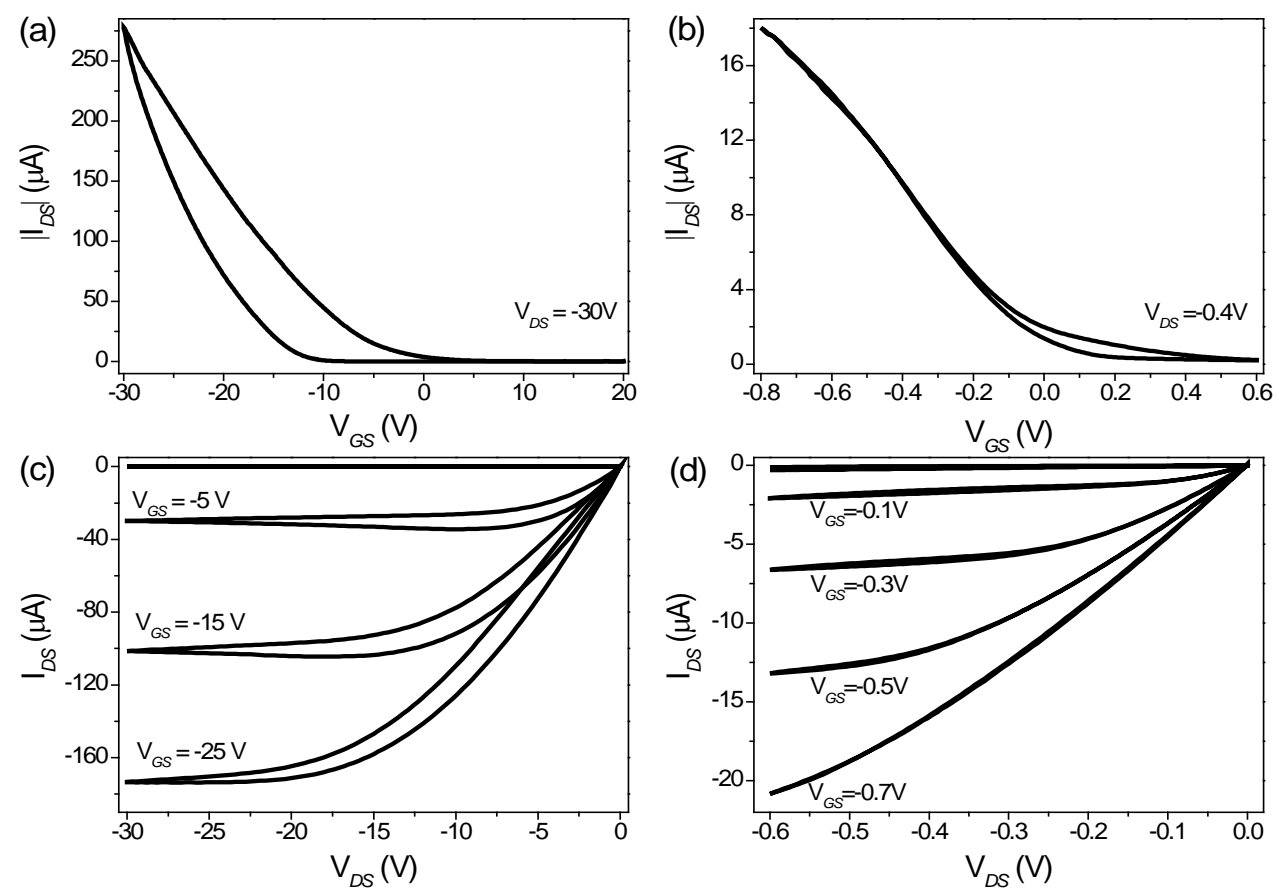

Figure S3 Electrical performance of PDPP-TT(1)-SVS(9):PS100K blend in the OFET and EGOFET configuration. OFET transfer (a) and output (c) characteristics, EGOFET transfer (b) and output (d) characteristics. 


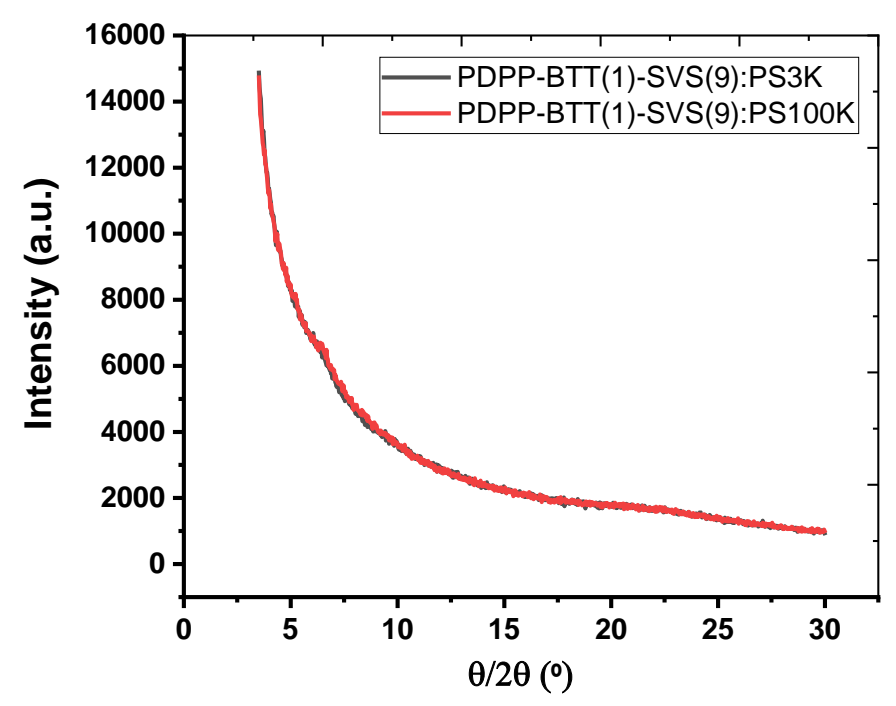

Figure S4 XRD result of PDPP-BTT(1)-SVS(9):polymer blends processed with polystyrene of two different molecular weights, i.e. Mw $3.000 \mathrm{~g} \mathrm{~mol}^{-1}$ (PS3K) and $100.000 \mathrm{~g} \mathrm{~mol}^{-1}$ (PS 100K).
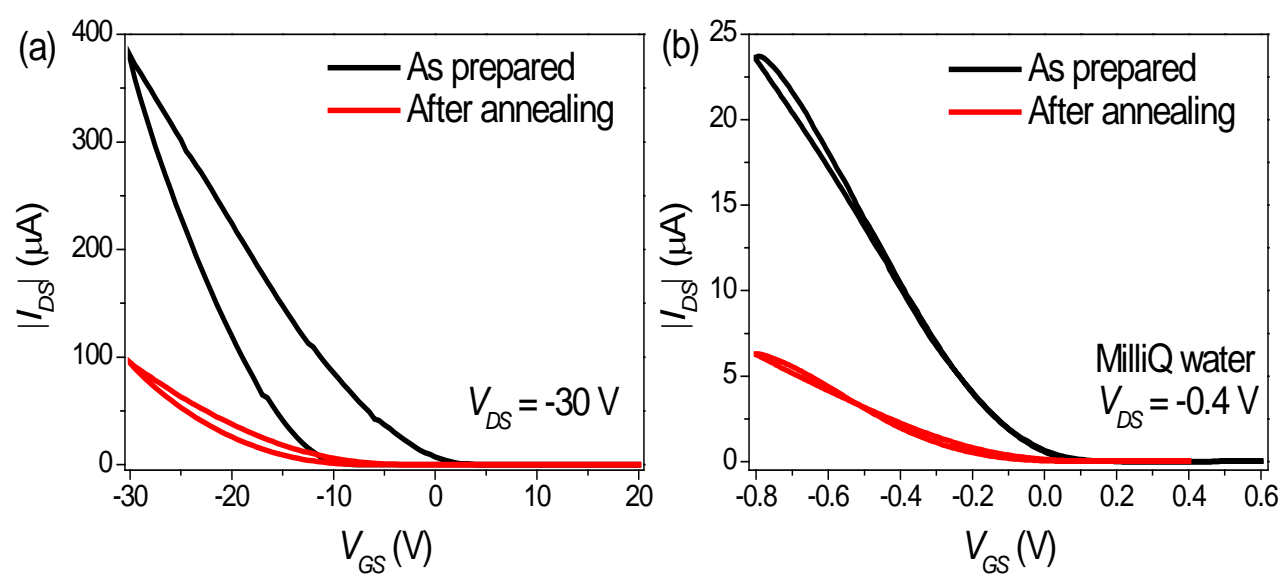

Figure S5 Electrical performance of PDPP-TT(1)-SVS(9):PS3K measured as prepared and after annealing as (a) OFET and (b) as EGOFET in the saturation regime. The annealing process was carried out in ambient condition by putting the device on the hotplate at $150{ }^{\circ} \mathrm{C}$ for 10 minutes. 

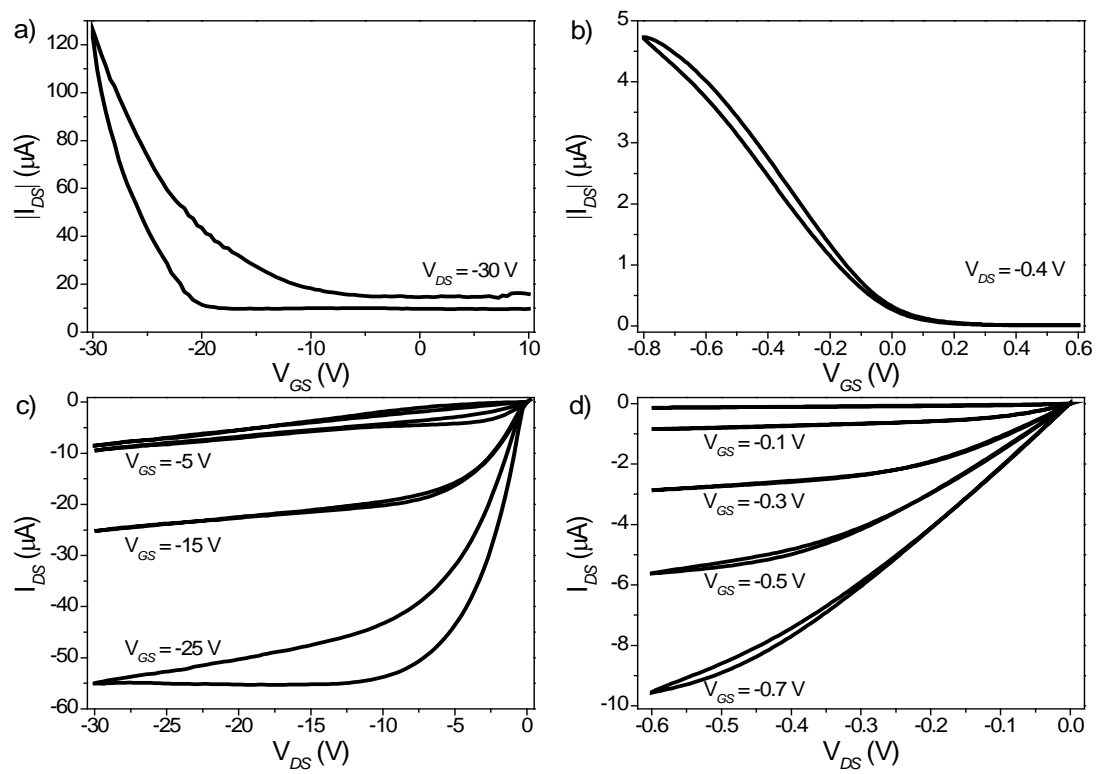

Figure S6 OFET a), c) and EGOFET b), d) electrical characteristics measured on the pristine PDPP-BTT(1)-SVS(9) thin film.
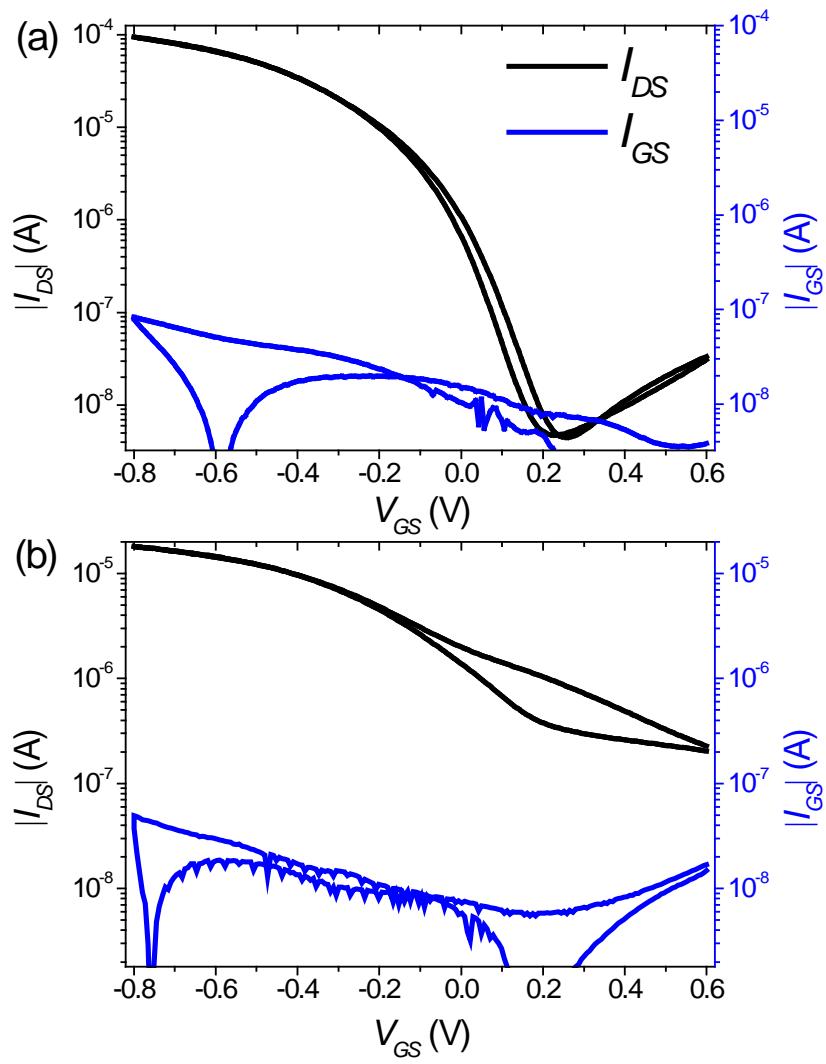

Figure S7 The drain-source (left axis) and gate-source (right axis) current of (a) PDPP-TT(1)SVS(9):PS3K and b) PDPP-TT(1)-SVS(9):PS100K measured as EGOFET device using MilliQ water as media in saturation regime $\left(V_{D S}=-0.4 \mathrm{~V}\right)$. 

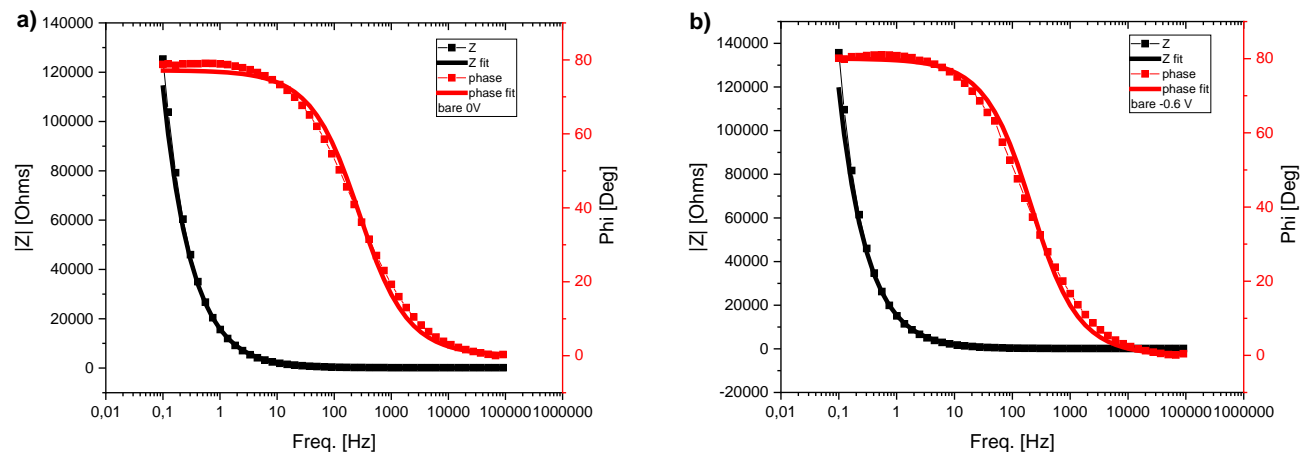

Figure S8 Nyquist Plot of the bare surface employed for the electrochemical analysis of the two thin film. The impedance has been recorded at (a) $V_{D C}=0 \mathrm{~V}$ and (b) $V_{D C}=-0.6 \mathrm{~V}$.

a)

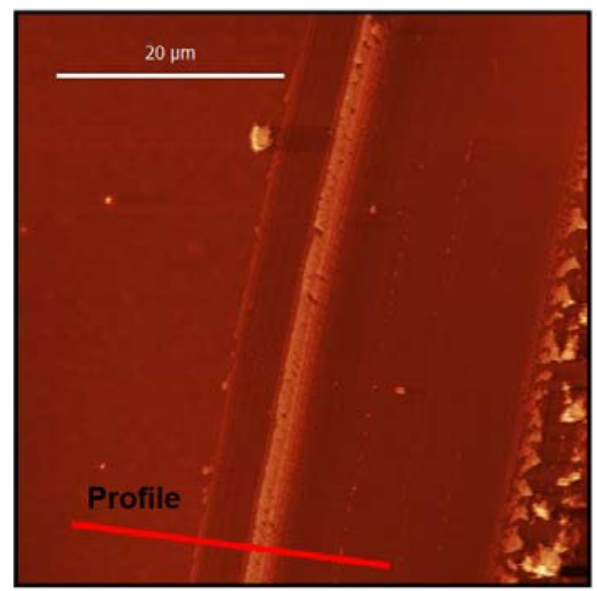

c)

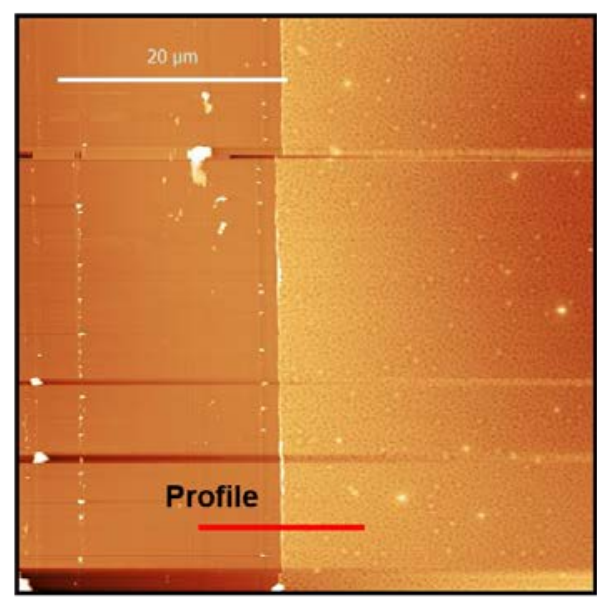

b)

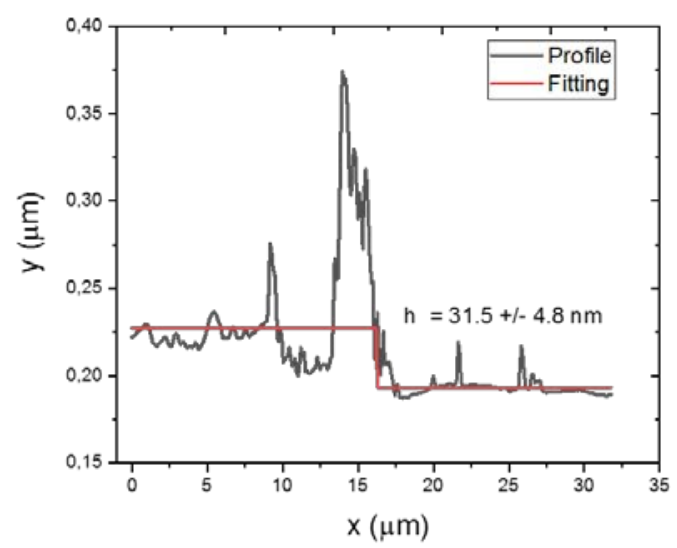

d)

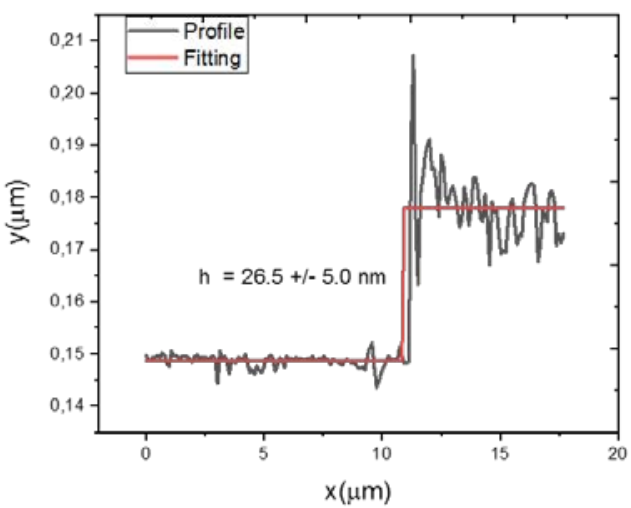

Figure S9 Height profiles extracted for (a and b) SVS:PS3K and (c and d) SVS:PS100K. 
a)

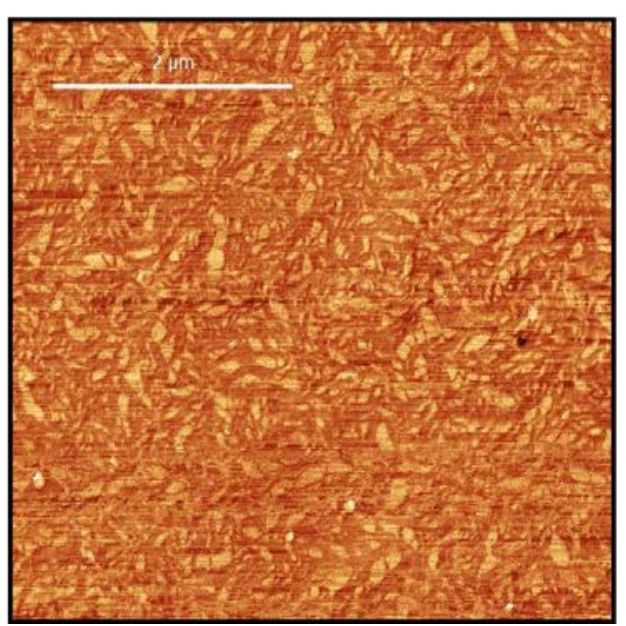

b)

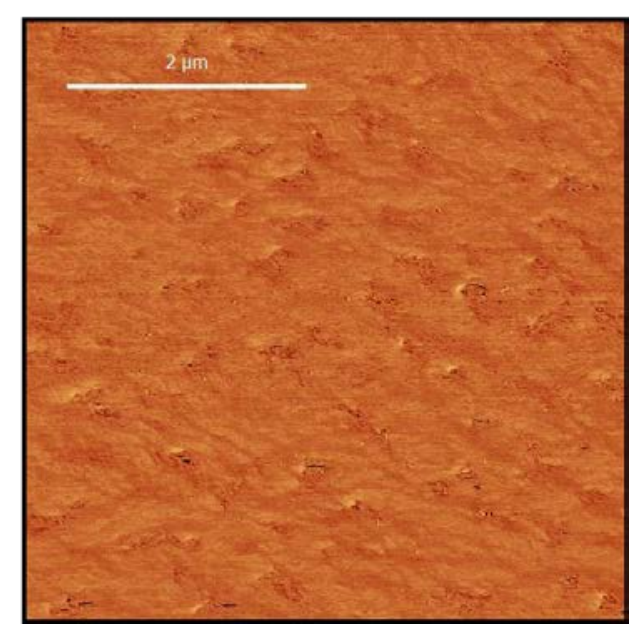

Figure S10 Phase images ( $\left.5 \times 5 \mu^{2}\right)$ of (a) SVS:PS3K and (b) SVS:PS100K.

a)

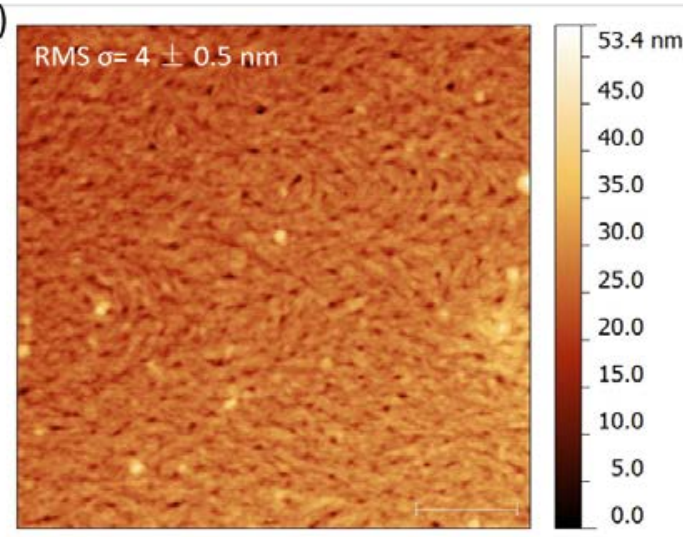

b)

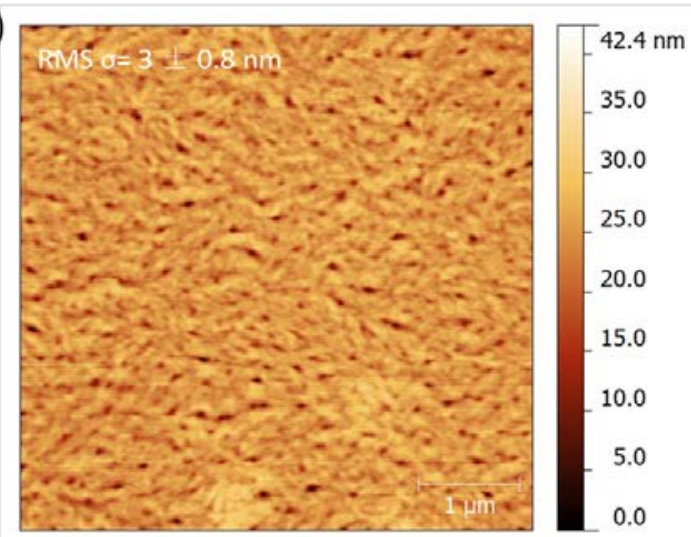

Figure S11 AFM images $\left(5 \times 5 \mathrm{~m}^{2}\right)$ of pristine PDPP-TT(1)-SVS(9) thin film on (a) Au electrode and (b) $\mathrm{SiO}_{2}$ channel region. The rms values are averaged from 5 images.

Table S1 The parameters extracted from the OFET and EGOFET configuration based on the two blends.

\begin{tabular}{|c|c|c|c|c|c|c|}
\hline \multicolumn{7}{|c|}{ PDPP-TT(1)-SVS(9):PS3K } \\
\hline \multicolumn{5}{|c|}{ OFET } & \multicolumn{2}{|c|}{ EGOFET } \\
\hline Device & $\mu\left(\mathrm{cm}^{2} \mathrm{~V}^{-1} \mathrm{~s}^{-1}\right)$ & $V_{t h}(\mathrm{~V})$ & $I_{o n} / I_{o f f}$ & $\mu C\left(\mu \mathrm{F} \mathrm{V}^{-1-1-1}\right)$ & $V_{t h}(\mathrm{mV})$ & $I_{\text {on }} / I_{\text {off }}$ \\
\hline 1\# & 0.31 & 3.8 & $1.1 \times 10^{7}$ & 0.33 & -93 & $\begin{array}{l}I_{\text {on }}^{\prime} \times \text { off } \\
1.0 \times 10^{4}\end{array}$ \\
\hline 2\# & 0.26 & 2.1 & $9.1 \times 10^{6}$ & 0.45 & -59 & $2.1 \times 10^{4}$ \\
\hline 3\# & 0.23 & 6.6 & $1.6 \times 10^{7}$ & 0.21 & 35 & $1.5 \times 10^{4}$ \\
\hline 4\# & 0.20 & 1.5 & $2.6 \times 10^{6}$ & 0.21 & 43 & $1.3 \times 10^{3}$ \\
\hline $5 \#$ & 0.18 & -4.5 & $4.1 \times 10^{6}$ & 0.26 & 107 & $6.0 \times 10^{3}$ \\
\hline 6\# & 0.12 & 5.5 & $3.6 \times 10^{6}$ & 0.28 & 41 & $1.7 \times 10^{4}$ \\
\hline Average & 0.22 & & & 0.33 & & \\
\hline \multicolumn{7}{|c|}{ PDPP-TT(1)-SVS(9):PS100K } \\
\hline $1 \#$ & 0.16 & 0.2 & $6.9 \times 10^{6}$ & 0.15 & 30 & $6.6 \times 10^{2}$ \\
\hline 2\# & 0.12 & -5.1 & $4.6 \times 10^{6}$ & 0.15 & 15 & $3.1 \times 10^{3}$ \\
\hline 3\# & 0.06 & 6.1 & $8.0 \times 10^{5}$ & 0.15 & 12 & $6.1 \times 10^{3}$ \\
\hline 4\# & 0.06 & 6.0 & $2.1 \times 10^{5}$ & 0.12 & 12 & $1.2 \times 10^{3}$ \\
\hline $5 \#$ & 0.07 & -3.3 & $4.0 \times 10^{6}$ & 0.12 & 19 & $7.9 \times 10^{3}$ \\
\hline Average & 0.10 & & & 0.15 & & \\
\hline
\end{tabular}

